\title{
Octogenarians and nonagenarians with severe symptomatic and asymptomatic carotid disease: does older age indicate "high risk" for carotid endarterectomy?
}

\author{
Franco Mazzalai*1, Oreste Terranova ${ }^{1}$, Mario Gruppo ${ }^{1}$, Giorgio Meneghetti ${ }^{2}$, \\ Claudio Baracchini ${ }^{2}$ and Enzo Ballotta ${ }^{1}$
}

Address: ${ }^{1}$ Department of Surgical and Gastroenterological Sciences, University of Padua, Italy and ${ }^{2}$ Department of Neurosciences, University of Padua, Italy

* Corresponding author

\author{
from XXI Annual Meeting of The Italian Society of Geriatric Surgery \\ Terni, Italy. 4-6 December 2008 \\ Published: I April 2009 \\ BMC Geriatrics 2009, 9(SuppI I):A4 doi:10.1 186/I47I-23I8-9-SI-A4
}

This abstract is available from: http://www.biomedcentral.com/I47I-23 I8/9/SI/A4

(C) 2009 Mazzalai et al; licensee BioMed Central Ltd.

\section{Background}

Patients older than 80 years old were excluded from participation in the large controlled randomized trials that demonstrated the efficacy of carotid endarterectomy (CEA) in preventing stroke in selected symptomatic and asymptomatic patients. Because of limited longevity and perceived increased perioperative risk from CEA, alternative treatment options, such as carotid angioplasty and stenting (CAS) have been suggested as a lower-risk alternatives. Many of the reports evaluating CAS have thus used age older than 80 years as one of the high-risk criteria allowing entry into the studies. We analyzed 30-day stroke and death rates after CEA in patients aged 80 or more with severe symptomatic and asymptomatic carotid disease.

\section{Patients and methods}

A retrospective review was conducted on a prospectively compiled computerized database of all primary CEAs performed by a single surgeon at our institution from 1990 to 2007. Descriptive demographic data, risk factors, surgical details, perioperative strokes and deaths, and other complications were recorded.

\section{Results}

In all, 1689 CEAs were performed in 1500 patients; 171 were performed in 159 patients aged $\geq 80$, (group I) and 1518 in 1341 patients <80 years old (group II). All CEA procedures involved either traditional CEA with patching
( $n=302)$ or eversion CEA $(n=1387)$ and were performed with patients under deep general anesthesia and cerebral protection involving continuous perioperative EEG monitoring for selective shunting. No strokes or deaths occurred in the group I, whereas there were 11 perioperative strokes and 3 deaths in the group II, for a stroke and death rates of $0.7 \%$ and $0.2 \%$, respectively.

\section{Conclusion}

The conviction that older age means higher risk needs to be revised. Patients aged $\geq 80$ can undergo CEA with no more perioperative risks than younger patients. Age older than 80 years should no longer be used as an inclusion criterion for entry into CAS trials. 